\title{
Chronic ankle instability: the value of talar tilt under general anaesthesia
}

\author{
A. W. McCaskie FRCS*, D. W. Gale FRCS ${ }^{\dagger}$, D. Finlay MRCP FRCR and M. J. Allen FRCS* \\ ${ }^{*}$ Glenfield General Hospital, Leicester LE3 9QP, UK, ' County Hospital, Lincoln LN2 5QY, UK, Leicester Royal \\ Infirmary, Leicester, UK
}

\begin{abstract}
Ankle inversion injuries are the most common injury in sport and yet treatment is controversial. One result is chronic ankle instability, an injury for which criteria for surgical intervention are unclear. This prospective study of 14 patients showed that a large proportion of patients had no detectable abnormality on preoperative investigation, but were subsequently shown to have an abnormality under general anaesthesia. This suggests that a good history of chronic instability is more sensitive an indicator than conventional investigations. Stress views under anaesthesia may confirm the problem.
\end{abstract}

Keywords: chronic ankle instability, surgical repair, talar tilt, general anaesthesia

Ankle inversion injuries are the most common injury in sport and yet the treatment of ankle sprains remains controversial ${ }^{1}$. Chronic ankle instability can be the result, which can benefit from operative correction. Stress talar tilt testing without general anaesthesia is often used to demonstrate the injury but can be inaccurate. The clinical criteria for surgical intervention remain unclear. The purpose of this preliminary study was to evaluate talar tilt under general anaesthesia as a diagnostic test for ankle instability.

\section{Method}

A prospective study was undertaken. 14 patients with chronic ankle instability were included in the study. Each patient had been investigated with plain radiographs of the ankle (antero-posterior and lateral), and with an arthrogram. During the arthrogram stress views of talar tilt were performed. The patients were accepted for surgical correction on clinical grounds. Following the administration of a general anaesthetic, including muscle relaxant, but prior to surgery, a further stress talar tilt was performed on both sides.

Address for correspondence: Mr A. W. McCaskie, University Department of Orthopaedic Surgery, Glenfield General Hospital, Groby Road, Leicester LE3 9QP, UK

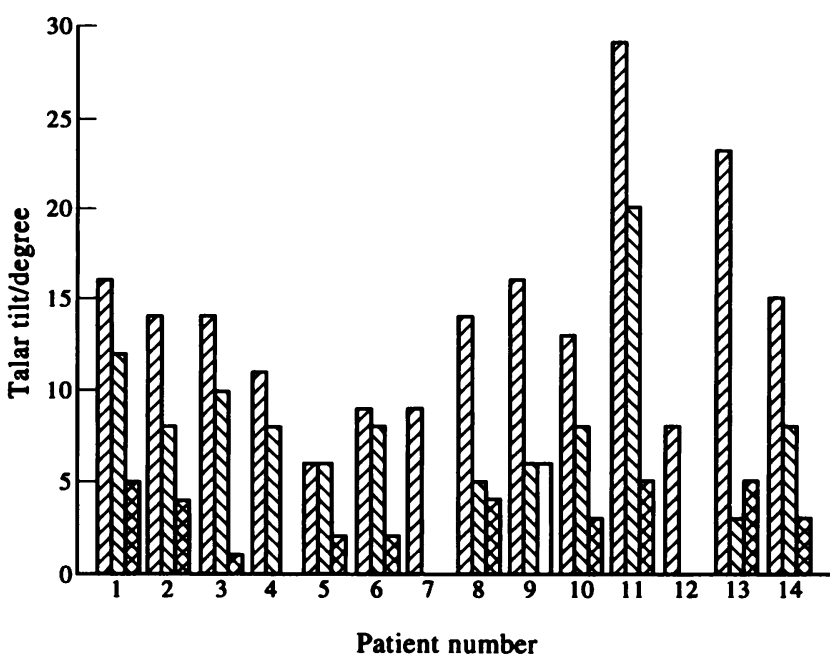

Figure 1. Stress talar tilt for all patients under different testing situations $(\mathbb{Z}$ G.A., Arthrogram, Control)

\section{Results}

Radiographic analysis was carried out on 14 patients. 10 patients were female and 4 male. The mean age was 31.7 years (range 14-57 years). The talar tilt was determined for: the injured side at arthrography, the injured side at operation, and finally the uninjured side at operation (control). The mean values of talar tilt were 7.2 degrees, 13.6 degrees, and 2.9 degrees respectively. Figure 1 illustrates the three measurements in all patients. The arthrogram was positive for capsular tear in only $50 \%$ of the patients.

\section{Discussion}

The value of talar tilt that should be considered abnormal is unknown. In a study of 202 naval midshipmen only $1.7 \%$ had a talar tilt of over $5^{\circ 2}$. Another diagnostic abnormality states that the talar tilt angle on the injured side must be more than twice that of the uninjured side (or have a value greater than $\left.9^{\circ}\right)^{3}$. If the arthrogram stress test group is considered first, using the first definition, 10 of the 14 
were abnormal, and using the second definition only 8 out of the 14 were abnormal. If the general anaesthetic stress group is considered next, using the first definition 13 of the 14 were abnormal, and using the second definition all the values were abnormal. The reader is reminded that only 7 of the 14 had abnormal arthrogram results. The results described in this study show that for some patients an abnormality cannot be demonstrated preoperatively. However, this group does show an abnormality on G.A. stress films. Such findings lead us to conclude that, firstly, accurate history taking is of great importance in the assessment of chronic ankle instability and that secondly, a failure to demonstrate an abnormality using an arthrogram and standard stress views does not exclude surgical repair from a patient in which it is clinically indicated. Furthermore, it may be useful to perform stress talar tilt views under anaesthesia before proceeding to reconstruction as a way of demonstrating the abnormality.

\section{References}

1 Lassiter TE, Malone TR, Garrett WE. Injury to the lateral ligaments of the ankle. Orthopaedic Clinics of North America 1989; 20: $629-40$.

2 Cox JS, Hewes TF. 'Normal' talar tilt angle. Clin Orthop 1979; 140: 37-41.

3 Kelikian H, Kelikian AS. Disorders of the Ankle. Philadelphia, WB Saunders, 1985, 447. 